\title{
PARENTAL SATISFACTION REGARDING TWO TYPES OF COMMERCIAL CROWNS FOR RESTORING PULPOTOMIZED PRIMARY MOLARS
}

\author{
Nehal Raid Salman*, Nagwa Khattab ${ }^{* *}$, Yaser Gomaa*** and Ahmad Elheeny ${ }^{* * * *}$
}

\begin{abstract}
Background: Restoration of pulpotomized primary molars with esthetic restorations becomes a priority in pediatric dentistry.

Aim: The purpose of this study was to assess parental satisfaction towards prefabricated zirconia crowns.

Methods: A randomized controlled trial included 60 children; pulpotomized mandibular right second primary molars restored with prefabricated zirconia crowns and mandibular left second primary molar restored with stainless steel crowns. On a 5 point-Likert-scale, a questionnaire was used to assess parental satisfaction was evaluated by parents at 12 months.
\end{abstract}

Results: Parental satisfaction with the treatment was excellent; however, satisfaction with regard to color received the highest significant rating (100\%) for zirconia crowns compared to stainless steel crowns. Parents rated their overall satisfaction in the size, shape and durability after 12 months from baseline. The ratings were (50.8\%) for size, shape and durability for both zirconia and stainless steel crowns.

Conclusion: Zirconia crowns had more esthetic parental satisfaction than stainless steel crowns.

KEYWORDS: Pediatric; Esthetic; Crown.

\section{INTRODUCTION}

In pediatric dentistry practice, restoration of pulpotomized primary molars is a regular procedure (1). One of the determining variables in the success or failure of pulpotomized treated primary molars is the use of a proper final restoration ${ }^{(2)}$. Restoration of pulpotomized primary molars restore lost tooth structure while also improving mechanical and functional qualities, improving the tooth's long-

\footnotetext{
* Assistant Lecturer, Pediatric Dentistry, Faculty of Dentistry, Pharos university, Alexandria, Egypt

** Professor, Department of Pediatric Dentistry and Dental Public Health, Faculty of Dentistry, Ain Shams University, Egypt

*** Professor, Department of Dental Biomaterials, Faculty of Dentistry, Minia University, Egypt

**** Associate Professor, Pediatric and Community Dentistry, Faculty of Dentistry, Minia University, Egypt.
} 
term prognosis and durability ${ }^{(3)}$. In order to please both patients and parents, esthetic restoration are frequently required ${ }^{(4)}$. As a result, transitioning from SSCs and toward more esthetic alternative restorations has become a focus. Parents' happiness with their child's postoperative appearance and smile must be considered by the clinician. There have been studies in the literature that challenge parents' satisfaction with zirconia crowns and SSCs for their children in terms of the degree of color match, general contour or failure rate ${ }^{(4-7)}$.

For primary molars, a range of commercially esthetic crowns are available, such as veneered SSC, NuSmile (Houston, TX, USA), Kinder Krowns (St Louis Park, MN, USA), Ez Crowns (Sprig Oral Health Technologies) and Cheng Crown (Exton, PA, USA) ${ }^{(6)}$. Prefabricated zirconia crowns of zirconia-toughened alumina type Y-TZP emerged in 2008 for anterior and posterior primary dentition. Their mechanical features as superior fracture resistance, are equivalent to metal crown properties, aside from their superior esthetic properties and low thermal conductivity ${ }^{(7)}$. Zirconia is a crystalline dioxide of zirconium that has metal-like mechanical characteristics, and its color is similar to that of teeth. Ready-made zirconia crowns are now available for both, primary incisors and molars ${ }^{(8)}$.

For primary molars, an adequate reduction is a determinant factor of passive fitting. The tooth reduction necessary for zirconia crowns is slightly more extreme than that required for SSCs by 20$30 \%{ }^{(9)}$. The purpose of the current study was to evaluate parental satisfaction of zirconia crowns for the treatment of mandibular primary molars and compare it with the SSC.

\section{SUBJECTS AND METHODS}

\section{Ethical considerations}

The study was reviewed and approved by an Institutional Review Board (Record number \#186). Informed consent was obtained from all children's parents/guardians.

\section{Sample calculation, design and setting}

The study was designed as a randomized controlled trial. Sample size calculation depended on the clinical success rate of zirconia crowns in previous literature. The sample size was calculated on a two-sample, one group with Zirconia and one control group (SSC) by effect size of proportion's difference of parents' satisfaction $26 \%$ (4). The inclusion of 25 crowns in each group would be sufficient to detect a statistically significant difference between interventions at a significance level of 5\% with a power of 80. To compensate for dropouts, 5 additional patients were included in each group leading to a total of 30 children for each group.

\section{Study Setting}

Sixty subjects were selected from the outpatient clinic in Pediatric and Community Dentistry department, Faculty of Dentistry, Minia University. The patient had bilateral mandibular molars requiring crowns.

\section{Subjects selected fulfilled the following criteria:}

1. Age range of the patients from 4 to 7 years old.

2. Have no history of spontaneous pain or swelling.

3. Patients willing to return for follow-up examinations and evaluation.

4. Medically free patients or with controlled systemic disease ASA I or II.

5. No active periodontal diseases.

6. Patients with posterior pulpotomized tooth/teeth indicated for crown restoration (e.g. 2-3mm of teeth above cement-enamel junction).

While, Molars should fulfil the following criteria:

1. Molars with MOD cavities or large multisurface cavities.

2. Molars with proximal caries extending to the line angles. 
3. No periapical, radicular lesions nor root resorption present in preoperative radiographic examination in molars.

\section{Exclusion criteria}

1. Patients with bad oral hygiene.

2. Uncooperative patients.

3. Patients with psychiatric problems or unrealistic expectation.

4. Patients with parafunctional habits (clenching/ bruxism).

\section{Randomization, allocation and masking}

The eligible children were randomly included in the study using computer generated block randomization list. Allocation was performed using properly sealed opaque envelope with treatment codes then was delivered by a resident in Pediatric Dentistry department who is completely blinded of the randomization code.

\section{Clinical steps}

For each child, 2 visits were required. In the first visit pulpotomy and zirconia crown preparation were performed for $2^{\text {nd }}$ primary right molar and crown on one side was cemented. Then, pulpotomy and SSCs procedure and preparations was performed on the $2^{\text {nd }}$ primary left molar in the second visit on the contralateral side.

\section{Pulpotomy}

After anaesthetizing the child with articaine hydrochloric $4 \%$ with epinephrine 1:100,000 (Septocaine ${ }^{\circledR} \quad 1.7 \mathrm{ml}, \quad$ SEPTODONT Ltd) and rubber dam isolation, caries was removed and access to the pulp chamber was gained by removal of its roof using \# 558 non-end cutting bur under copious water coolant spray. Coronal pulp tissues were removed with sharp, sterile excavator. A piece of cotton soaked with formocresol (Sultan, USA) was inserted into the pulp chamber for 3 minutes.
After removing the formocresol pellet, a thick mix of polymer reinforced Zinc-oxide/Eugenol (ZOE) (Zinconol, Prevest DenPro. India) paste was packed into the pulp chamber to seal the orifices. Self-cured glass ionomer (GIC) (Riva self-cured, Australia) was applied over the capping ZOE.

\section{Tooth preparation for zirconia crowns}

1. Occlusal preparation using the marginal ridge of the adjacent teeth as a reference point, using coarse flame diamond bur, 1.5-2 mm of Occlusal reduction was performed on primary lower right $2^{\text {nd }}$ molar following the natural contour of the existing clinical crown. An adequate occlusal reduction is extremely important for the proper fit and placement of pediatric zirconia crowns. The final occlusal plane of the seated pediatric zirconia crown was determined by the amount of occlusal reduction.

2. Buccal and lingual surfaces reduced with a minimum of $0.5-1.0 \mathrm{~mm}$ of tooth structure.

3. Mesial and distal slices were made proximally, tapered to a knife /feather edge at the gingival margin. Interproximal areas were opened, and the crown dimensions were reduced by $20-30 \%$ (or $0.5-1.25 \mathrm{~mm}$ ) using a tapered diamond bur $^{(11)}$.

4. At this stage, it is easiest to complete a full circumferential reduction approximately $15 \%$ to $20 \%$ using the same bur supragingivally to visualize the completeness and evenness of the preparation. The prepared tooth reduction was extended from 1 to $2 \mathrm{~mm}$ subgingivally. A subgingival preparation was achieved using a more tapered and finer diamond bur. A full subgingival reduction to approximately $1.5 \mathrm{~mm}$ depth was attained, ensuring a smooth, ledgefree feather-edged margin. The prepared tooth walls were finished with a 1-2 $\mathrm{mm}$ subgingival feather-edge preparation using a thinner pointed tapered diamond bur ${ }^{(12)}$. 


\section{Crown selection for zirconia crowns}

1. An appropriate prefabricated crown size was selected before the tooth preparation ${ }^{(12)}$. The mesiodistal dimension was measured by a caliper and selection of the size from the trial fit kit crowns to be used based on the size of the molar. After tooth preparation, right side was allocated to receive pink try-in crowns for the NuSmile pediatric crown (Orthodontic Technologies Inc., Houston, TX, USA) ${ }^{(13,14)}$ to check preparation, size, fit and occlusion. Then the final crown size was selected after preparation. Any necessary refinements to the preparations would be done at this point and occlusion was checked again. Also, pulpal therapy would be completed at this point if needed, and the try-in crowns would be checked for fit after such treatment.

2. Each prepared tooth was washed and dried, but not desiccated. The final passive fit of the final crown was confirmed and cemented with G-CEM ${ }^{\mathrm{TM}}$ capsules self-adhesive resin cement (GC Corporation, Tokyo, Japan) mixed as directed by the manufacturer ${ }^{(15)}$. Crowns were allowed to set for 24 hours according to the manufacturer's instructions into the correct position and excess cement was wiped from visible surfaces. Ask the patient to hold the crown under occlusal compression until luting cement setting using cotton roll.

3. Excess cement was then cleaned and flossed interproximally. Once all excess cement was satisfactorily removed.

4. The occlusion was checked and the patient was advised to come for regular check-ups.

\section{Tooth preparation for stainless steel crowns}

1. Occlusal surface was reduced by $1-1.5 \mathrm{~mm}$ with a diamond wheel bur (TeezKa-van Ltd, Tehran, Iran).

2. Proximal undercuts were reduced almost vertically using featheredge bur (TeezKavan Ltd, Tehran, Iran).
3. The occlusal third of the buccal surface was bevelled using a wheel bur.

4. Linear angles were rounded. Proper size SSCs of the primary lower left 2nd molars (3M ESPE, St. Paul, MA), were chosen. The crowns were fitted, contoured, crimped (no. 114, 3M ESPE, and no. 800-417, Denovo, Baldwin Park, CA) and controlled by the tip of an explorer in order to achieve the best marginal adaptation.

5. The crowns were then filled with glass ionomer cement (GC America, Inc., Alsip, IL, USA) in such a way that two-thirds of the internal surface of the crown and all margins were covered with the cement.

6. The crown containing cement was seated on the tooth and patient bite on a cotton roll until completion of the setting of the cement.

7. All procedures, including tooth preparation, selection, adjustment and cementation of crowns were performed by a single operator (pedodontist). In addition, the mixing of cement was performed according to the manufacturer's instructions ${ }^{(16)}$

\section{Adherence improvement session took place in the initial visit}

1. Each patient was informed about the study steps and maintain oral hygiene. Subsequent sessions occurred at the follow-up visits.

2. The patients were trained for tooth brushing, flossing and instructed with oral hygiene methods after each meal ${ }^{(17)}$.

Follow-up to patients were done at 6 and 12 months. Parents were administered a written questionnaire. Parents were asked to score parameters such as the crown's color, size, durability, and their overall satisfaction during the follow-up visit after 12 months from baseline. These characteristics of the crowns were scored using a scale of 1 to 5 , with 1 being "very unsatisfied" and 5 being "very satisfied"(20,21) Durability was defined as the crown's 
capacity to adhere on the tooth without cracking and its ability to function properly. The questionnaire was explained to the attending parent by a qualified dental assistant. The dental assistant documented the parent's responses, which were given verbally. The dentist was not present during the parent review, and parents were told that their responses would remain private. The parents evaluated their child's restoration directly and not from a photograph. The examiner was present to answer any queries the parents had about the survey. The survey asked about ethnicity/race, education level and other sociodemographic factors. The questionnaire included questions regarding parent's satisfaction of the color, form, durability and size of each crown. Parents were asked to score their overall experience ${ }^{(18)}$. In order to establish the parental opinion of the influence of the crowns, questions were asked about the child's oral health, esthetics, patient reaction to the crowns before and after intraoral insertion ${ }^{(20)}$. Responses were recorded on a Likert scale score of one to five, with one being not at all and five being very much. Parents were asked if they would recommend these crowns to another parent whose child needs posterior crowns (not recommend, recommend, or highly recommend) ${ }^{(18,19)}$. Parents were then asked to choose from a list of possible crown problems their children might have had, such as bleeding around the gums when brushing, food lodged between the crowns, and sensitivity to hot/ cold foods (more than one response was allowed). Finally, parents were given the chance to provide their thoughts about the crowns.

\section{RESULTS}

\section{Statistical analysis of the data}

Data were fed to the computer and analysed using IBM SPSS software package version 20.0. (Armonk, NY: IBM Corp), Comparisons between two groups for categorical variables were assessed using Chi-square test. Significance of the obtained results was judged at the $5 \%$ level.
TABLE (1): Comparison between parent's satisfaction regarding both crowns.

\begin{tabular}{ccccc}
\hline $\begin{array}{c}\text { Parent } \\
\text { satisfaction }\end{array}$ & $\begin{array}{c}\text { SSC } \\
(\mathbf{n}=\mathbf{6 0})\end{array}$ & $\begin{array}{c}\text { Zirconia } \\
\text { crowns } \\
(\mathbf{n}=\mathbf{6 0})\end{array}$ & $\chi^{\mathbf{2}}$ & $\mathbf{p}$ \\
\hline Size & & & & \\
4 & $30(50.8 \%)$ & $30(50.8 \%)$ & 0.000 & 1.000 \\
5 & $30(49.2 \%)$ & $30(49.2 \%)$ & & \\
Shape & & & & \\
4 & $30(50.8 \%)$ & $30(50.8 \%)$ & 0.000 & 1.000 \\
5 & $30(49.2 \%)$ & $30(49.2 \%)$ & & \\
Color & $31(50.8 \%)$ & $0(0 \%)$ & & \\
1 & $30(49.2 \%)$ & $0(0 \%)$ & $122.0^{*}$ & $<0.001^{*}$ \\
2 & $0(0 \%)$ & $60(100 \%)$ & & \\
5 & & & & \\
Durability & & & & \\
4 & $30(49.2 \%)$ & $30(49.2 \%)$ & 0.000 & 1.000 \\
5 & $30(50.8 \%)$ & $30(50.8 \%)$ & & \\
\hline
\end{tabular}

$$
\begin{aligned}
& \chi 2: \text { Chi square test } \\
& *: \text { Statistically significant at } p \leq 0.05
\end{aligned}
$$

\section{DISCUSSION}

The problem of dental decay in children's teeth is a major public health issue. There are several approaches for offering full coverage restoration for the primary dentition, each with its own set of benefits and drawbacks.

Today, esthetics has become a popular topic in dentistry. It is extremely important for a child's entire health and psychological well-being ${ }^{(21)}$. In recent years, esthetic crowns for primary molars have become available on the market, which would be a valuable asset if they could maintain the benefits of traditional stainless steel crowns. ${ }^{(22)}$.

There has been a wide selection of crowns accessible that meet the satisfaction and acceptance of parents in terms of both looks and function ${ }^{(20)}$. Aesthetics in very young children is a topic that has gotten a lot of interest in psychology literature. However, few research in the dental literature have sought to investigate aesthetics from the perspective of parents for primary posterior teeth ${ }^{(23)}$. 
As a result, the current study was conducted to assess parent satisfaction with two commonly used commercially available crowns. A five-point Likert scale was used for evaluation and the parents were asked to score parameters such as the crown's color, size, durability, and their overall satisfaction during the follow-up visit after 12 months from baseline. The dental assistant answered the questionnaire without the presence of the dentist. This was done to eliminate the risk that the dentist's presence would have put pressure on parents to give higher evaluations.

Parents were not pushed into giving higher scale ratings, according to the findings. Although a high degree of parental satisfaction was reported in a similar study examining prefabricated resinfaced stainless steel crowns, the lowest level of satisfaction was indicated for the crown's aesthetics (24). Parents reported higher statistically significant color satisfaction with zirconia crowns than with SSCs in the current trial. The shape, size and durability of the zirconia crown and SSCs, on the other hand, were not statistically significant. This discovery could be explained by the parent's freedom to choose the esthetic type of crown, resulting in high color satisfaction. The natural appearance of the zirconia crown, as well as its highly beautiful attributes, including translucency. No cracks, chips, fractures, or material loss were noted by either parents or dental evaluators when it came to crown durability. During the trial, just one crown debonded, and it happened two months after it was placed owing to a cement-to-tooth failure. With no further complications, an identical crown was placed. The current study's findings on parental satisfaction with ZCs were consistent with previous findings of Salami et al. ${ }^{(25)}$ and Yanover et al. ${ }^{(26)}$

\section{Limitations}

- Despite the fact that choosing patients with positive behaviour was one of the inclusion criteria, a few children became resistant owing to the lengthy treatment.

- The high cost of zirconia crowns.

\section{CONCLUSIONS}

- Restoration of primary teeth with esthetic materials has an increasing demand of parents in the dental office.

- Clinically, zirconia crowns are comparable to SSCs and suit the esthetic desires of parents, particularly in terms of color.

- Parents would choose zirconia crowns again and also recommend them to a friend.

\section{Recommendations}

- Longer follow-up studies on zirconia crowns with inclusion of more evaluation parameters such as patients' satisfaction especially with high cost of zirconia crowns, ease of handling, as well as other variables.

\section{REFERENCES}

1. Kratunova E, Silva D. Pulp therapy for primary and immature permanent teeth: An overview. Gen Dent. 2018;66(6):30-8.

2. Kuo HY, Lin JR, Huang WH, Chiang ML. Clinical outcomes for primary molars treated by different types of pulpotomy: A retrospective cohort study. J Formos Med Assoc [Internet]. 2018;117(1):24-33. Available from: https://doi.org/10.1016/j.jfma.2017.02.010

3. Guelmann M , Shapira J , Silva D R, Fuks A B . Esthetic restorative options for pulpotomized primary molars: a review of literature. J Clin Pediatr Dent. 2011;36(2):123-6.

4. Article O. Parental Satisfaction With Pediatric Posterior Preformed Zirconia and Stainless Steel Crowns- a Comparative. 2020;(2):44-9.

5. Abuelniel G, Eltawil S. Clinical and Radiographic Evaluation of Stainless Steel versus Zirconia Crowns on Primary Molars: Randomized Controlled Trial. Egypt Dent J. 2018;64(2):977-89.

6. Anjana G, Darshana V. Zirconia crowns in pediatric dentistry. J Indian Dent Assoc. 2019;1(1):29-33.

7. Mittal GK, Verma A, Pahuja H, Agarwal S, Tomar H. Esthetic Crowns In Pediatric Dentistry: A review. 2016;3(5):1280-2. 
8. Daou EE. The Zirconia Ceramic : Strengths and Weaknesses. 2014;33-42.

9. Clark L, Wells M H, Harris E F . Comparison of Amount of Primary Tooth Reduction Required for Anterior and Posterior Zirconia and Stainless Steel Crowns. Pediatr Dent. 2016;38(1):42-7.

10. Elheeny AAH. Allium sativum extract as an irrigant in pulpectomy of primary molars: A 12-month short-term evaluation. Clin Exp Dent Res. 2019;5(4):420-6.

11. Al-Haj Ali S. In vitro comparison of marginal and internal fit between stainless steel crowns and esthetic crowns of primary molars using different luting cements. Dent Res J (Isfahan). 2019;16(6):366-71.

12. Cazaux SL, Hyon I, Prud T, Trutaud SD. Reminder of important clinical lesson Twenty-nine-month follow-up of a paediatric zirconia dental crown. 2017;2-7.

13. Information G. Technical Guide.1-2.

14. Crowns AP. Anterior Pediatric Crowns.

15. Befestigungszement S, Kapselform IN, Scellement CDE, En A, Zahnes PDES, Dente PDEL, et al. G-CEM Capsule G-Cem Capsule G-Cem Capsule G-Cem Capsule G-Cem Capsule G-Cem Capsule. 2019;5-6.

16. Ramazani N, Ranjbar M. Effect of tooth preparation on microleakage of stainless steel crowns placed on primary mandibular first molars with reduced mesiodistal dimension. J Dent Tehran Univ Med Sci. 2015;12(1):18-24.

17. Kara Z, Yilmaz Y. Assessment of oral hygiene and periodontal health around posterior primary molars after their restoration with various crown types. 2014.
18. Roberts C, Lee JY, Wright JT. Clinical evaluation of and parental satisfaction with resin-faced stainless steel crowns. Pediatr Dent. 2001;23(1):28-31.

19. Shah P V., Lee JY, Wright JT. Clinical success and parental satisfaction with anterior preveneered primary stainless steel crowns. Pediatr Dent. 2004;26(5):391-5.

20. Holsinger DM, Wells MH, Scarbecz M, Donaldson M. Clinical evaluation and parental satisfaction with pediatric Zirconia anterior crowns. Pediatr Dent. 2016;38(3):192-7.

21. Bhuvaneswaran M. Principles of smile design. J Conserv Dent. 2010 Oct;13(4):225-32.

22. Fuks AB, Ram D, Eidelman E. Clinical performance of esthetic posterior crowns in primary molars: A pilot study. Pediatr Dent. 1999;21(7):445-8.

23. Pani SC, Saffan A Al, Alhobail S, Salem F Bin, Alfuraih A, Altamimi M. Esthetic Concerns and Acceptability of Treatment Modalities in Primary Teeth : A Comparison between Children and Their Parents. 2016;2016.

24. Mathew MG, Roopa KB, Soni AJ, Khan MM, Kauser A. Evaluation of Clinical Success, Parental and Child Satisfaction of Stainless Steel Crowns and Zirconia Crowns in Primary Molars. J Fam Med Prim care. 2020 Mar;9(3):1418-23.

25. Salami A, Walia T, Bashiri R. Comparison of Parental Satisfaction with Three Tooth-Colored Full-Coronal Restorations in Primary Maxillary Incisors. J Clin Pediatr Dent. 2015;39(5):423-8.

26. Yanover L, Waggoner W, Kupietzky A, Moskovitz M, Tickotsky N. Parental and Dentist Satisfaction with Primary Anterior Zirconia Crowns: A Case Series Analysis. Children. 2021;8(6):451. 